\title{
85695 - A PRESENÇA DE MULTIMORBIDADE ESTÁ ASSOCIADA AO DESEMPENHO FÍSICO INADEQUADO EM IDOSOS COMUNITÁRIOS?
}

\author{
Pôster - Gerontologia
}

\author{
Janaina Rocha Niehues / Niehues, JR / Universidade Federal de Santa Catarina - UFSC; \\ Maria Eduarda da Costa / Costa, ME / Universidade Federal de Santa Catarina - UFSC; \\ Núbia Carelli Pereira de Avelar / Avelar, NCP / Universidade Federal de Santa Catarina - UFSC; \\ Ana Lúcia Danielewicz / Danielewicz, AL / Universidade Federal de Santa Catarina - UFSC
}

Introdução: $\mathrm{O}$ envelhecimento populacional é um dos maiores desafios na área da saúde, devido suas inúmeras implicações, nas quais destacam-se o aumento de doenças crônicas e a presença de multimorbidade, as quais predispõem ao comprometimento do desempenho físico ${ }^{1}$. Objetivo: Avaliar a associação entre a presença de multimorbidade e o desempenho físico de idosos comunitários. Métodos: Trata-se de um estudo de base domiciliar e delineamento transversal. A amostra aleatória foi composta por idosos com 60 anos ou mais de idade, não institucionalizados, cadastrados no sistema de informação em saúde da Atenção Básica de Balneário Arroio do Silva SC. Foi considerada como multimorbidade a coexistência de três ou mais doenças crônicas (doença na coluna, artrite/reumatismo, hipertensão, diabetes e osteoporose), com base na metodologia adotada pela Pesquisa Nacional de Saúde ${ }^{2}$. No desempenho físico, foi avaliada a força de membros inferiores por meio do teste de sentar e levantar da cadeira de cinco repetições (TSLC5'rep), a mobilidade através do Teste Timed Up and Go (TUG) e a velocidade de caminhar por meio do teste de Velocidade de Marcha Habitual (VMH). Os testes foram analisados dicotomizados (desempenho adequado ou inadequado) considerando-se pontos de corte estabelecidos. Houve aprovação pelo Comitê de Ética em Pesquisa com Seres Humanos da UFSC (CAAE no7776318.3.0000.0121). Foram realizadas análises descritivas (frequências relativas e IC95\%) e analíticas por meio do teste Qui-quadrado de Pearson, adotando-se valores de $\mathrm{p} \leq 0,05$ como significativamente estatísticos. Resultados: foram avaliados 211 idosos (55,4\% do sexo feminino e 44,5\% do sexo masculino). A prevalência de multimorbidade foi de 64,4\% (IC95\% 57,5; 70,9). Houve associação significativa entre a presença da multimordidade e o desempenho inadequado no TSLC5'rep $(\mathrm{p}=0.001)$, TUG $(p=0,023)$ e VMH $(p=0,014)$. Conclusões: A multimorbidade em idosos comunitários é uma condição comum e está associada positivamente ao desempenho inadequado nos testes de força de MMII, mobilidade física e velocidade da marcha.

Palavras-chave: desempenho físico funcional, idoso, multimorbidades.

Referências: 1. MELO LA et al. Fatores associados à multimorbidade em idosos: uma revisão integrativa da literatura. Rev. bras. geriatr. gerontol., Rio de Janeiro, vol. 22(1), e180154, 2019; 2. IBGE, Pesquisa Nacional de Saúde 2013: Ciclos de vida: Brasil e grandes regióes. Rio de Janeiro IBGE, Coordenação de Trabalho e Rendimento: 92 p. 2015. 